\title{
Suppressing g Modes in Shell Hydrogen-Burning $\delta$ Scuti Stars
}

\author{
P. A. Bradley, J. A. Guzik \\ Applied Physics Division, X-2, MS B220, Los Alamos National \\ Laboratory, Los Alamos, NM 87545, USA
}

For shell hydrogen-burning $\delta$ Scuti stars, many more unstable modes are predicted than are observed. For example, for $4 \mathrm{CVn}$ and $\delta$ Scuti itself, only 18 and 6 modes are observed, respectively, whereas several hundred $\ell=0,1$ and 2 rotationally-split modes are predicted. The predicted modes have a mixed p- and g-mode character, with many g-type nodes present in the H-exhausted core and shell H-burning region, where the Brunt-Väisälä frequency is large.

Here we explore whether the predicted frequency spectrum can be made to agree better with the observed spectrum if the g-mode character of the pulsation modes is partially suppressed. Additional motivation for this approach is provided by the observation by Breger et al. (1999) that 4 modes in 4 CVn identified as $\ell=1$ have nearly equal frequency spacings of $\sim 14 \mu \mathrm{Hz}$, reminiscent of consecutive radial orders from a pure p-mode spectrum. We consider 2.1- and 2.3- $M_{\odot}$ evolutionary models that match the observed $\mathrm{T}_{\text {eff }}, L$, and $\log g$, plus an identified radial mode frequency of $4 \mathrm{CVn}$ and $\delta$ Scuti. For these models, we experimented with setting the Brunt-Väisälä frequency to zero from the model center to the edge of or beyond $\mathrm{H}$-depleted core, so that the g-type portion of the waves becomes evanescent in this region.

We find that the frequency spacing agrees best with observations if the Brunt-Väisälä frequency is set to zero throughout the core and most of the $\mathrm{H}$-burning shell. In this case, the number of predicted modes in the observed frequency range is reduced by a factor of three, but many more modes are still predicted than observed.

More work is necessary to develop some physical justification for the possible suppression of core g-mode propagation. These stars are not predicted to have convective cores or shells which would damp g modes. Nonadiabatic calculations by Lee (1985) and Guzik (reported by Cox 1990) show that, unlike red giants and Cepheids with degenerate cores, the radiative dissipation in evolved $\delta$ Scuti stars is insufficient to prevent the formation of standing waves in the core.

\section{References}

Breger, M., Handler, G., Garrido, R., et al. 1999, A\&A, 349, 225

Cox, A. N. 1990, in Nonlinear Astrophysical Fluid Dynamics, eds. J.R. Buchler \& S.T. Gottesman, New York Acad. Sci., pp. 54-72

Lee, U. 1985, PASJ, 37, 279 